\title{
Left Ventricular Maximum Negative dP/dt
}

National Cancer Institute

\section{Source}

National Cancer Institute. Left Ventricular Maximum Negative dP/dt. NCI Thesaurus.

Code C120918.

The maximum rate of negative pressure (diastolic) change within the left ventricle during a unit time. 